\title{
COVID-19 and neurosurgical training and education: an Italian perspective
}

\author{
Cesare Zoia ${ }^{1}$ (1) - Giovanni Raffa ${ }^{2} \cdot$ Teresa Somma $^{3}$ - Giuseppe M. Della Pepa ${ }^{4}$ - Giuseppe La Rocca ${ }^{4,5} \cdot$ Matteo Zoli $^{6,7}$. \\ Daniele Bongetta ${ }^{8} \cdot$ Oreste De Divitiis $^{3} \cdot$ Marco M. Fontanella $^{9}$
}

Received: 31 May 2020 / Accepted: 11 June 2020 / Published online: 18 June 2020

(C) Springer-Verlag GmbH Austria, part of Springer Nature 2020

\begin{abstract}
Background The severe acute respiratory syndrome coronavirus 2 (SARS-CoV-2) pandemic has an impact also on neurosurgical training and education, especially in most affected countries. We surveyed Italian neurosurgical residents, asking them to provide a brief description and analyze the situation they are experiencing and how the educational system reacted to the pandemic in one of the most affected countries all over the world.

Methods An 18-question, web-based survey was administered to Italian neurosurgical residents from May 3 to May 11, 2020, by web-link or e-mail invitation. Closed-ended, multiple choice questions were focused on the experience of neurosurgical residents in the last 2 months (from March to May 2020) concerning both clinical and educational aspects.

Results Among 331 Italian neurosurgical residents invited to participate, 192 responded to the survey (58\%). According to the participants' responses, in the whole country, only $29.7 \%$ of residents were directly involved in the clinical management of COVID-19 patients. Time spent in the clinic and surgical activity was significantly reduced in most of the cases. Educational activities as well as scientific activity and time spent for studying, on the other hand, were reported to be significantly increased by the majority of respondents.

Conclusions Most Italian neurosurgical residents reported significant changes on both training and education, highlighting a prompt reaction of the educational system in the whole country, regardless the local and regional diffusion of the pandemic.
\end{abstract}

Keywords COVID-19 $\cdot$ Neurosurgical residents $\cdot$ Survey $\cdot$ Neurosurgery

This article is part of the Topical Collection on Neurosurgery Training

Electronic supplementary material The online version of this article (https://doi.org/10.1007/s00701-020-04460-0) contains supplementary material, which is available to authorized users.

Cesare Zoia

gioiaoffice@gmail.com

1 Department of Neurosurgery, Fondazione IRCCS Policlinico San Matteo, viale Golgi 19, 27100 Pavia, Italy

2 Division of Neurosurgery, BIOMORF Department, University of Messina, Messina, Italy

3 Department of Neurosciences and Reproductive and Odontostomatological Sciences, Division of Neurosurgery, Università degli Studi di Napoli Federico II, Naples, Italy

4 Institute of Neurosurgery, Fondazione Policlinico Universitario A. Gemelli IRCCS, Catholic University, Rome, Italy
Department of Neurosurgery, Mater Olbia Hospital, Olbia, Italy

6 Department of Neurosurgery, IRCCS Institute of Neurological Sciences of Bologna, Bologna, Italy

7 Department of Biomedical and Neuromotor Sciences, University of Bologna, Bologna, Italy

8 Department of Neurosurgery, ASST Fatebenefratelli Sacco, Milan, Italy

9 Division of Neurosurgery, Department of Surgical Specialties, Radiological Sciences and Public Health, University of Brescia, Brescia, Italy 


\section{Introduction}

The COVID-19 pandemic is not over yet and its consequences will be seen for a long time across the world. Apart from new scenarios that we will face in the next weeks, the pandemic has already deeply impacted the life of billion peoples around the world, leaving dramatic changes and sequelae in almost all the aspects of the social life. The medical community has been probably one of the most impacted sectors, especially because the tremendous efforts physicians and caregivers were required to afford to contain the spreading of the pandemic. Among this, also the Neurosurgical Community has been involved into the management of the pandemic, being asked to continue ensuring appropriate neurosurgical care also to COVID-19 patients. On the other hand, practice has consistently changed during the SARS-CoV-2 pandemic and neurosurgery also had to adapt, coming in the help of the national health systems, especially in the most affected countries like Italy, to manage COVID-19 patients outside the neurosurgical clinics $[3,6,7,9,14,19]$. Obviously, in such a dramatic scenario, also neurosurgical education and training have been influenced by the pandemic. Some papers have been already published on this topic $[1,2,4,10,12,18]$ but they reported just the authors' point of view and do not investigate the opinion of those directly involved. Conversely, reporting the point of view of residents who experienced the real and concrete changes of the neurosurgical education and training due to the pandemic in most affected countries could be extremely useful. That could serve as a useful feedback to educational providers, eventually suggesting adjustments of the current educational model during the pandemic. We, therefore, conducted an online survey that was submitted to neurosurgical residents in Italy, one of the most affected countries all over the world.

\section{Materials and methods}

An 18-question web-based anonymous survey was administered to Italian neurosurgical residents from May 3 to May 11, 2020, via the Google Forms platform.

The list of all neurosurgical residents in Italy was obtained from the universities' websites and all were invited to fill in the questionnaire. Residents were invited by sending them the survey web-link through e-mail and/or social networks. The questions were focused on the experience of neurosurgical residents in the last 2 months (from March to May 2020) and analyzed different clinical and educational aspects, including time spent in clinic in routine neurosurgical practice, time spent outside the neurosurgical clinic for the management of COVID-19 patients, availability of personal protective equipment (PPE), COVID-19 screening, and finally educational and scientific activities. All question were closed-ended, multiple choice (Supplementary Material). The primary goal was to collect data on neurosurgical residents' perceptions of the health emergency, the measures undertaken for them, and the changes in neurosurgical practice, training, and education. Data were elaborated using Google Forms software online and responses were analyzed considering the overall survey participants in a first step; thereafter, a further subgroup analysis has been performed distinguishing between junior (PGY-1 and 2) vs. senior (PGY-3, 4, and 5) residents.

\section{Results}

On May 11, 2020, the survey was closed after 192 responses out of the 331 Italian neurosurgical residents were received. Collectively, the $58 \%$ of the Italian neurosurgical residents participated to the survey. Residents from all the 5 postgraduate years (PGY) were homogenously represented: 41 PGY-1 (21.4\%), 43 PGY-2 (22.4\%), 31 PGY-3(16.1\%), 40 PGY-4 (20.8\%), 37 PGY-5 (19.3\%) (Fig. 1).

The first observed result was that no Residency Programs has been stopped in Italy, unlike the medical student's internship, which has been stopped to preserve student health and to limit the number of people present in the neurosurgical departments.

Resident's time spent in neurosurgical departments was reduced in 139 cases $(72.4 \%)$, unchanged in 40 cases $(20.8 \%)$, and increased only in 13 cases $(6.8 \%)$. This "worktime" reduction was imposed by the educational providers in $63.5 \%$ of cases, to avoid unnecessary exposure of residents to COVID-19 patients (Fig. 2). The majority of responders (135, $70.4 \%$ ) were not directly involved in the treatment of "COVID-19" patients (inside or outside neurosurgical department); the other 57 managed "COVID-19" patients under supervision $(42,21.9 \%)$ or alone $(15,7.8 \%)$. The majority of responders $(72.9 \%)$ answered that they did not receive any theoretical preparation on the use of PPE, and the 55.2\% said that the given PPEs were not adequate to their duties, at least at the beginning of the pandemic. Residents involved in the direct management of "COVID-19" patients, both inside and outside the neurosurgical departments, reported to have received good support from supervisors most of the time $(65.9 \%)$. The overall reported screening rate for SARSCoV-2 between residents was $35.9 \%$; Sixteen $(8.3 \%)$ were tested in March and 53 (27.6\%) in April/May, while 123 (64.1\%) did not perform any screening test. Thanks probably also to the reduction of the working time, $83.9 \%$ of residents did not refer any symptoms of the COVID-19 infection. Almost all of the interviewed reduced their surgical activity (78.6\% perform less operation and $16.1 \%$ did not perform any operation at all); only 3 participants reported their surgical activity was increased, while 7 reported an unchanged surgical 
Fig. 1 Post-graduate year

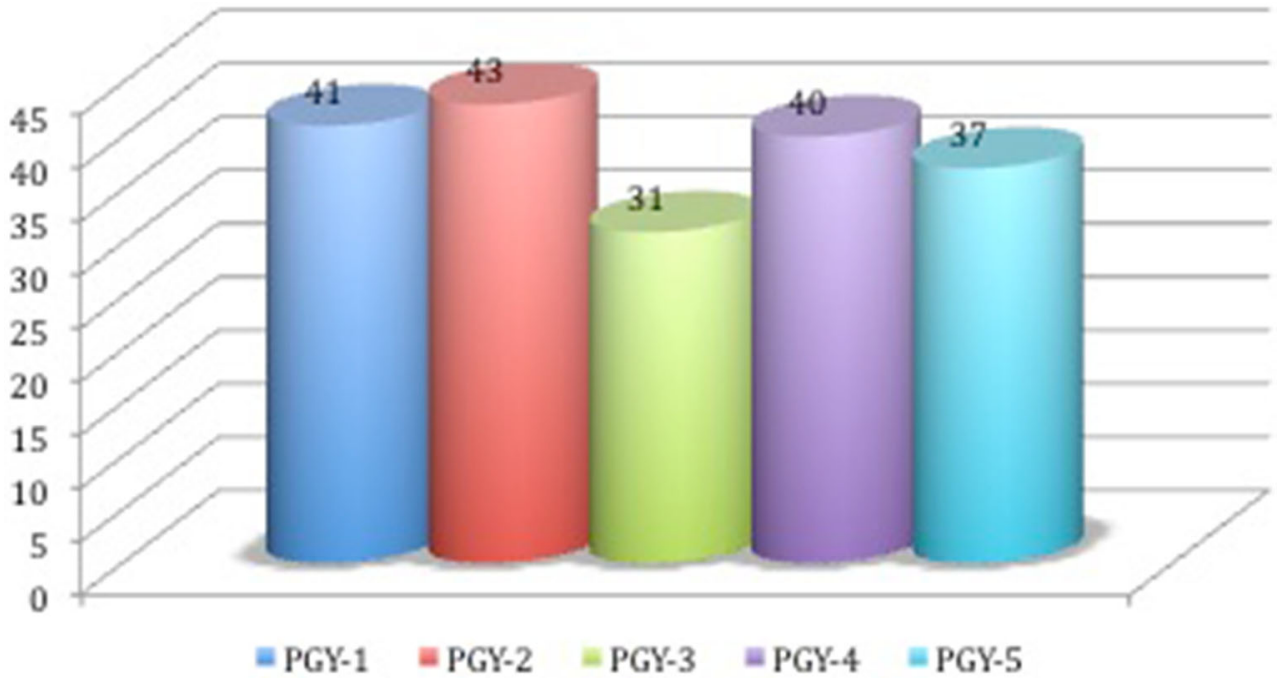

activity (Fig. 3). In cases of neurosurgical procedures performed on "COVID-19" patients, participants responded their engagement was unchanged compared to usual in $59.1 \%$ of cases, reduced in $39.1 \%$, and augmented in $1.8 \%$. According to the responders' opinion, the contribution of residents (not only neurosurgical ones) in emergency management was fundamental $(42.7 \%)$ or useful $(40.6 \%)$ in supporting healthcare providers. Finally, participants homogenously reported an increase of the educational and scientific activities, as well as of time spent for studying. The production of scientific papers or research projects was increased in $55.7 \%$ of cases and also the theoretic educational activity had an increase for $64.6 \%$ of responders (Fig. 4). That was mainly due to the spread and increased availability and visibility of neurosurgical seminars and webinars performed though different web-based commercial platforms, and strongly promoted by the educational

\section{Have you had a reduction in working hours/attendanceat the clinic?}

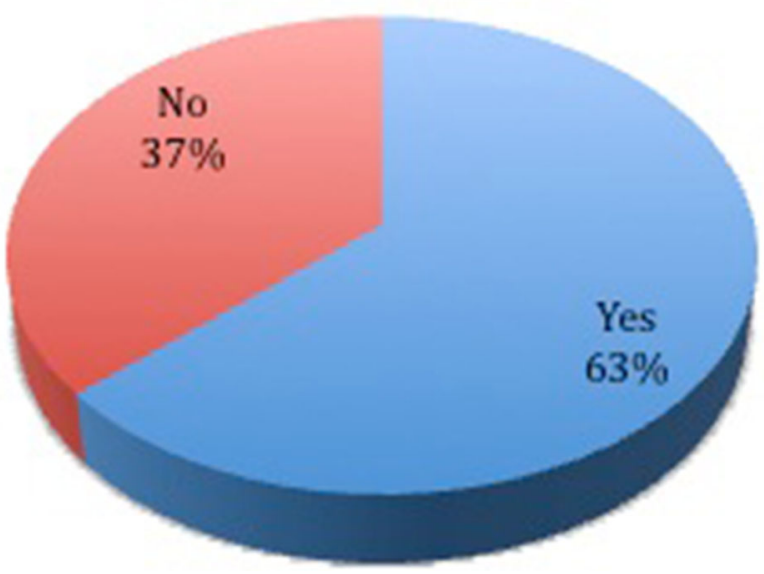

Fig. 2 Reduction in working time providers. Online educational activity was carried out mainly on a national basis $(86.5 \%)$, through a wide network of several Residency Programs, and not only on a local basis. Finally, the majority of responders $(71.9 \%)$ affirmed their time spent for studying was increased in the last 2 months, probably because of the reduced working time at neurosurgical departments. The subgroup analysis of responses according to the PGY showed that no major differences were reported by senior vs. junior residents in almost all the survey questions. Interestingly, an evident difference was noticed for questions regarding surgical and scientific activity. In particular, among $78.6 \%$ (151 out of 192 responders) of participants who reported a reduced surgical activity, senior resident represented the $80.1 \%$ (121 out of 151). Similarly, senior residents reported a more evident increase of their scientific activity as well as of time spent for studying compared with the junior ones. In particular, senior residents accounted for the $73 \%$ (78 out of 107 ) and the $69 \%$ (95 out of 138) of participants who reported respectively an increase of scientific activity and time spent for studying during the last 2 months.

\section{Discussion}

Also, many areas of the modern society, including neurosurgical care, have been dramatically impacted and changed by the COVID-19 pandemic; also, the fundamental sector of neurosurgical training and education suffered from deep changes and limitations. Despite experts' and educational providers' point of view on the topic being already reported [10-12], the most appropriate way to assess how neurosurgical education and training has changed in the last 2 months still remains to hear from opinions of directly involved people, neurosurgical residents.

In Italy, that has been for long time the most affected country in the world (before being sadly overpassed by Spain and, 
Fig. 3 Reduction in surgical activity
Your surgical activity in the past 2 months is

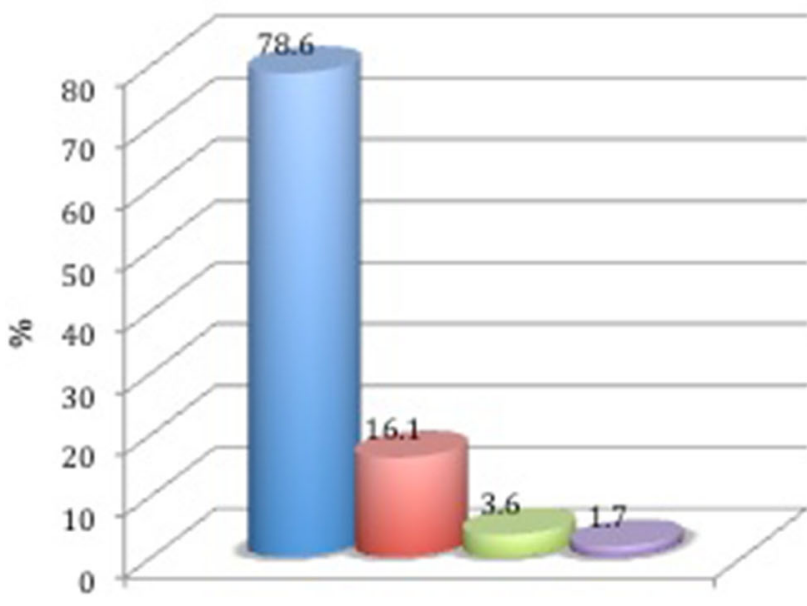

Decreased

I I haven't operated in the past two months

Unchanged

increased lastly, the USA), neurosurgical residents felt on their own the impact of the COVID-19 pandemic.

First of all, our survey demonstrated that neurosurgical educational providers promptly reacted to the pandemic: the first result was that no Residency Programs were stopped but there was a tendency to reduce the working time of neurosurgical residents to avoid unnecessary exposure to the COVID19. In any case, when needed, some residents were asked to help the National Health System in the management of COVID-19 patients, even outside Neurosurgical Departments. Residents answered responsibly to their duty and supported healthcare providers in the management of COVID-19 patients, waiving to their neurosurgical practice, education, and training (at least during their clinical working time).

Since the spread of the virus was very different along the Italian peninsula, elective neurosurgical activity decreased in all Italian regions and collectively, we recorded a reduction of the residents' working time at neurosurgical departments in the majority of responses $(63.5 \%)$. That, of course, mainly impacted surgical practice, especially because at the same time also the activity in the Operating Room (OR) was limited to emergent and oncologic cases in many hospitals of the country.

Sadly, the sudden onset of the COVID-19 outbreak in Italy combined with the complex and chaotic international trading of PPE reduced the availability of PPE for all healthcare providers including neurosurgical residents, especially during the first weeks from the beginning of the emergency; that is witnessed by the response to the survey in which $55.2 \%$ of responders said that available PPEs were not adequate to their duties.

The reduced time spent at neurosurgical departments and for surgical practice was translated in an increase of educational and scientific activities, as well as of time spent for individual study. Educational providers revealed to pay a great
Fig. 4 Increase in theoretical activity

\section{The theoretical activity(lessons, seminars, journal clubs, ...) in the last two months is}

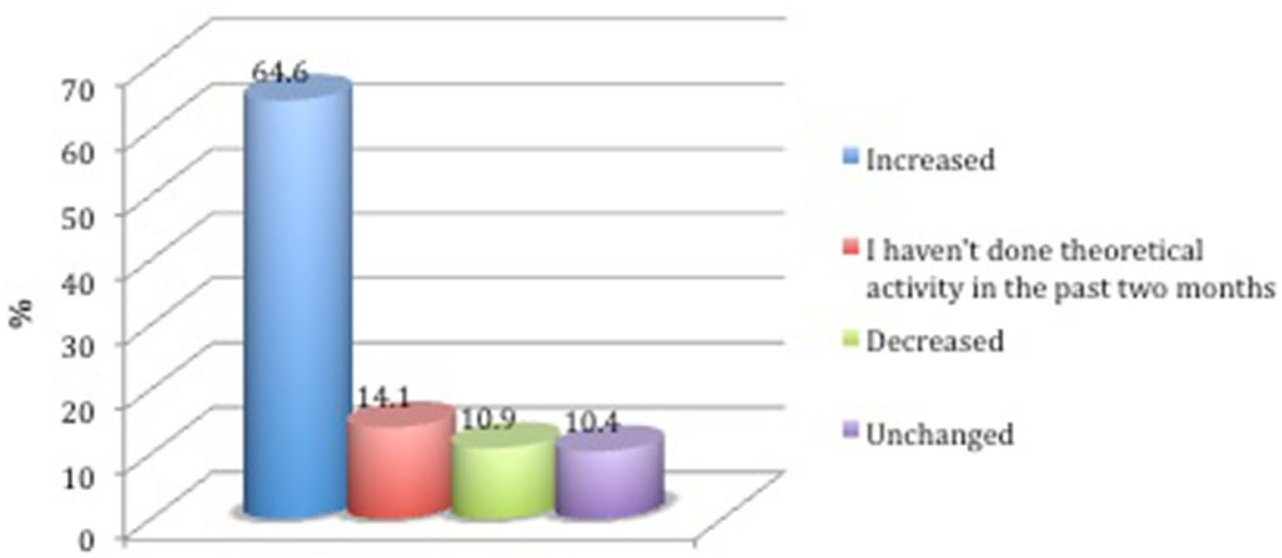


attention to this topic and tried to promptly compensate the reduced practice in the OR with an increase of the offer for online activities like seminars, webinars, and frontal lessons. That resulted in a homogenous positive feedback from survey participants, who reported an increase of the web-based educational activity (64.6\%), even through the participation to a nation-wide network of neurosurgical residency programs providing frontal lessons, expert opinions, and discussions on neurosurgical hot topics (reported by the $86.5 \%$ of survey participants). Similarly, the increased time availability outside neurosurgical departments resulted in an increased scientific activity of residents (55.7\% of responders), as well as a longer time spent to individual study as compared the pre-COVID-19 era (71.9\% of participants).

Interestingly, we observed a different responses' trend among senior vs. junior residents regarding changes in surgical, scientific, and study activities. Senior residents were more affected by the global reduction of neurosurgical activity as compared with PGY-1 and 2 residents, probably because they are usually more involved in direct surgical training than their younger colleagues. Conversely, their reduced time spent in the OR was compensated with an increase of time spent for study and especially for writing research projects, scientific drafts, and papers. As they should have already maturated good scientific attitudes and skills in comparison with junior residents, they have been able to exploit the reduced time spent in surgical training for a deeper commitment to scientific and study activities.

Despite these few differences between senior and junior residents, all participant's responses collectively highlight the fact that, apart the dramatic impact of the pandemic resulting, in some cases, in their direct involvement in the clinical management of COVID-19 patients outside neurosurgical departments and the reduction of their neurosurgical practice, the pandemic caused also the enhancement of the web-based neurosurgical education. Online neurosurgical education on national and/or international basis has been already strongly advocated prior to the COVID-19 outbreak as a formidable tool to increase neurosurgical knowledge and improve patient care, as well as to reduce the worldwide gap in neurosurgical education [5]. Nevertheless, many national neurosurgical societies all over the world do not possess an adequate web-visibility to promote online neurosurgical education $[8,17]$. In Italy, neurosurgical educational providers were incredibly able to re-organize their teaching model in few weeks, creating new opportunities for online neurosurgical education on a local basis but also at a nation-wide level, thanks also to the contribution and support of the Italian Society of Neurosurgery (SINch) that already had a good web-visibility prior to the COVID-19 outbreak. We are sure that such a new online nation-wide network for neurosurgical education will continue to offer new learning opportunities even after the end of the COVID-19 crisis, thus further enriching the educational opportunities for Italian neurosurgical residents.

While there may not yet be other similar studies of neurosurgery resident experience during COVID-19 with which to compare the results of this study, it is possible to compare findings from our study with those from other studies regarding different Residency Programs. Marasco et al. described findings from a survey distributed among young Italian gastroenterologists: they reported a reduced activity of gastroenterology units that was restricted to emergency visits and endoscopy, with reductions of activities of up to $90 \%$. Moreover, $84.5 \%$ of participants felt that the COVID-19 outbreak impacted on their training, due to unavailability of mentors $(52.6 \%)$ and interruption of trainee's involvement (66.4\%) [13]. These findings are similar to our results, especially for the global reduction of the clinical/surgical activity. Nevertheless, it is worth to notice that the survey by Marasco et al. did not mentioned about the implementation of novel web-based models for assuring residents' and young physicians' education. Conversely, the organization of new web-based educational networks represents the most important novelty for neurosurgical training introduced after the COVID-19 outbreak.

Another useful comparison can be done with the organizational model of the orthopedic residency program reported by Schwartz et al., who proposed to divide residents into two teams, the "active-duty inpatient" and "remotely-working," which remained completely isolated from each other to decrease their exposure to the virus $[15,16]$. This method seems to be really effective to maintain residents' surgical practice and training, as well as to guarantee appropriate surgical care to patients. Nevertheless, in countries where such a model cannot be easily and quickly implemented, the Italian neurosurgical model based on the global reduction of the residency surgical training and its compensation with improved scientific and online educational activities seems a good compromise to protect residents from contagion while ensuring their theoretical education.

Our study has some limits. First, it is not an epidemiologic study and does not allow drawing conclusions about the actual prevalence and incidence of the variables investigated. It does allow, though, drawing conclusions regarding the perception of Italian neurosurgical residents about the COVID-19 health emergency. Moreover, another limitation is that the survey is not validated. The typical process for development of a psychometric instrument involves searching the literature to see what is currently available and in use, focus groups and interviews to decide on topics, the development of individual questions, and then testing and validation of the instrument prior to distribution. This helps maximize the validity and reliability of the instrument. In the case of the present study, such a process cannot be performed because of the necessity to develop the survey and distribute it as quickly as possible, given the 
overall pandemic situation. Notwithstanding, this is the first survey conducted on the impact of COVID-19 on the neurosurgical residents about their training and education, and we believe that the data from this study can help to understand the impact of this pandemic in these specific fields.

\section{Conclusions}

The spread of COVID-19 has put new obstacles to neurosurgical training, especially in most affected countries. Italian educational models and teaching methods have been forced to change in few weeks, demonstrating a really effective response to the pandemic outbreak through the use of modern web-based tools. Despite Italian neurosurgical residents facing a transient reduction of their surgical training, they had the possibility to compensate this lack with increased opportunities for theoretical education, thanks also to the creation of educational networks at a local and nation-wide level. "Not all evil comes to harm": the enhancement of online education is for sure a good opportunity that this pandemic will leave to Italian neurosurgical residents.

\section{References}

1. Bambakidis NC, Tomei KL (2020) Editorial. Impact of COVID-19 on neurosurgery resident training and education. J Neurosurg. https://doi.org/10.3171/2020.3.jns20965

2. Bray DP, Stricsek GP, Malcolm J, Gutierrez J, Greven A, Barrow DL, Rodts GE, Gary MF, Refai D (2020) Letter: maintaining neurosurgical resident education and safety during the COVID-19 pandemic. Neurosurgery. https://doi.org/10.1093/neuros/nyaa164

3. Cenzato M, DiMeco F, Fontanella M, Locatelli D, Servadei F (2020) Editorial. Neurosurgery in the storm of COVID-19: suggestions from the Lombardy region, Italy (ex malo bonum). J Neurosurg:1-2

4. Clark VE (2020) Editorial. Impact of COVID-19 on neurosurgery resident research training. J Neurosurg. https://doi.org/10.3171/ 2020.4.jns201034

5. Downes SR, Lykina T (2020) Closing the gap in global neurosurgical education via online conference: a pre-COVID survey. Cureus. https://doi.org/10.7759/cureus.8015

6. Fontanella MM, De Maria L, Zanin L et al (2020) Neurosurgical practice during the severe acute respiratory syndrome coronavirus 2 (SARS-CoV-2) pandemic: a worldwide survey. World Neurosurg. https://doi.org/10.1016/j.wneu.2020.04.204

7. Fontanella MM, Saraceno G, Lei T, Bederson JB, You N, Rubiano AM, Hutchinson P, Wiemeijer-Timmer F, Servadei F (2020)
Neurosurgical activity during COVID-19 pandemic: an expert opinion from China, South Korea, Italy, United States of America, Colombia and United Kingdom. J Neurosurg Sci. https://doi.org/10.23736/S0390-5616.20.04994-2

8. Germanò A, Scibilia A, Raffa G, Esposito F (2018) Websitevisibility of neurosurgical centers in Europe. A necessary tool for enhancing scientific network cooperation and information distribution: letter to the editor. Acta Neurochir 160:1493-1495

9. Germanò A, Raffa G, Angileri FF, Cardali SM, Tomasello F (2020) Coronavirus disease 2019 (COVID-19) and neurosurgery: literature and neurosurgical societies recommendations update. World Neurosurg. https://doi.org/10.1016/j.wneu.2020.04.181

10. Harary M, Bergsneider M (2020) Letter: approaches to mitigate impact of COVID-19 pandemic on neurosurgical residency application cycle. Neurosurgery. https://doi.org/10.1093/neuros/ nyaa176

11. Hu YJ, Min ZJ, Ping CZ (2020) Experiences of practicing surgical neuro-oncology during the COVID-19 pandemic. J Neuro-Oncol. https://doi.org/10.1007/s1 1060-020-03489-6

12. Khosravi MH, Sisakht AM, Kiani D, Ahmadi S (2020) Effects of COVID-19 pandemic on neurological surgery care and education: our experience from Iran. World Neurosurg. https://doi.org/10. 1016/j.wneu.2020.05.058

13. Marasco G, Nardone OM, Maida M, Boskoski I, Pastorelli L, Scaldaferri F (2020) Impact of COVID-19 outbreak on clinical practice and training of young gastroenterologists: a European survey. Dig Liver Dis 157(5):310-314

14. Perin A, Servadei F, DiMeco F et al (2020) May we deliver neurooncology in difficult times (e.g. COVID-19)? J Neurooncol. https:// doi.org/10.1007/s11060-020-03496-7

15. Ramos O, Mierke A, Eastin M, Morrison M, Wongworawat DM, Danisa O (2020) COVID-19 pandemic and the implications for orthopaedic and neurosurgery residents and fellows on spine rotations. North Am Spine Soc J. https://doi.org/10.1016/j.xnsj.2020. 100006

16. Schwartz AM, Wilson JM, Boden SD, Moore TJ, Bradbury TL, Fletcher ND (2020) Managing resident workforce and education during the COVID-19 pandemic. JBJS Open Access. https://doi. org/10.2106/jbjs.oa.20.00045

17. Scibilia A, Raffa G, Priola SM, Esposito F, Angileri FF, Cardali SM, Germanò A (2019) Neurosurgery on the web: an analysis of the web-visibility of the European Neurosurgical Societies. J Neurosurg Sci. https://doi.org/10.23736/S0390-5616.19.04637-X

18. Theofanis TN, Khanna O, Stefanelli A, Al Saiegh F, Farrell C, Jabbour P, Harrop JS, Rosenwasser RH, Sharan A (2020) Letter: neurosurgery residency in the COVID-19 era: experiences and insights from Thomas Jefferson University Hospital, Philadelphia, Pennsylvania. Neurosurgery. https://doi.org/10.1093/neuros/ nyaa211

19. Zoia C, Bongetta D, Veiceschi P, Cenzato M, Di Meco F, Locatelli D, Boeris D, Fontanella MM (2020) Neurosurgery during the COVID-19 pandemic: update from Lombardy, northern Italy. Acta Neurochir. https://doi.org/10.1007/s00701-020-04305-w

Publisher's note Springer Nature remains neutral with regard to jurisdictional claims in published maps and institutional affiliations. 